\title{
COMMENT
}

Check for updates

\section{Medical images, social media and consent}

Jonathan P. Segal(10 ${ }^{1}$ and Richard Hansen $\mathbb{1}^{2 \times}$

The popularity of social media amongst medical professionals has led to widespread use for both networking and education. Limited professional guidance exists on the sharing of medical imagery on these platforms. This Comment explores consent and offers reflective advice on the use of medical images on social media.

Medical images have always had an extended role, beyond their clinical purpose, to help educate professionals. Historically, the sharing of medical images has been predominantly reserved through professional avenues in the format of lectures, textbooks and medical journals. With the explosion of the internet, and now social media, the dissemination of information is changing rapidly, and sometimes unrecognisably. Social media platforms have become an increasing part of many healthcare professionals working lives, arguably more so with the impact of social distancing during the COVID-19 pandemic and the expansion of video conferencing for clinical activity ${ }^{1}$. There has been a corresponding increase in the use of virtual platforms for professional networking and medical education ${ }^{2}$, leading to the emergence of highly specialised and often sensitive discussions within a public forum in a way never seen before in traditionally closed medical conferences or other formal educational settings. This change has led to the sharing of patient imagery on these platforms, often in short-hand form, to aid the telling of clinical stories, to highlight a teaching point, or to ask for peer advice or support. A study published in 2021 described the landscape of Twitter posts in gastrointestinal endoscopy. Walradt and colleagues ${ }^{3}$ identified 356 gastroenterologists active on Twitter, of whom $24.1 \%$ posted images or videos of endoscopy across 956 tweets. Importantly, $18.5 \%$ of these tweets were identified as "at-risk" for confidentiality non-compliance, including patients' dates of birth and even on occasion the patient's face; these were distributed across $31.3 \%$ of the gastroenterologists sharing such content. A further $1.4 \%$ of tweets were considered non-compliant. Only a single tweet described as "at-risk" discussed patient consent.

The issue of consent is an important one. Whilst the Walradt et al. paper ${ }^{3}$ offers a largely North American perspective and points to the useful Health Insurance Portability and Accountability Act that helped define "protected health information" ${ }^{4}$, here we offer a UK-based commentary. As already noted, medical images on social media are often provided without specific written consent and increasingly made available on a publicly accessible platform. The UK regulator, the General Medical Council (GMC), highlights that the standards expected of doctors do not change because they are communicating through social media, hence doctors need to be mindful of the content they post and engage with in order to maintain this professionalism ${ }^{5}$. The GMC is clear that maintenance of patient confidentiality is one of the pillars of the profession that every clinician must adhere to. In the UK, the Caldicott Principles stipulate considerations when sharing potentially confidential information ${ }^{6}$ : "justify the purpose(s); do not use personal confidential data unless it is absolutely necessary; use the minimum necessary personal confidential data; access to personal confidential data should be on a strict need-to-know basis; everyone with access to personal confidential data should be aware of their responsibilities; comply with the law; the duty to share information can be as important as the duty to protect patient confidentiality".

The rules that govern the sharing of medical imaging have been well defined and stipulate that when images or material are potentially identifiable through the image itself, the accompanying text or clinical story, then written consent is required from the patient and this stance is supported by the $\mathrm{GMC}^{5,6}$. In the situation in which data are anonymised or coded, the GMC states that these can be used for research, teaching, training or any health-care-related purpose without consent. There is, however, a lack of clarity about how this guidance relates to the expanding use of such images on social media. Importantly, these broad principles have long been followed by medical journals when publishing medical images in both their print copy and corresponding online content ${ }^{7}$. Despite this, it is important to reflect that consent for an image in a scholarly publication might not extend to consent for these images to be presented on social media in a public forum.

In accordance with the principles of consent, a patient must first have capacity to agree to sharing their clinical image, it must be voluntary, and the patient should 


\section{Box 1 | Clinical image sharing on social media}

- Consider seeking support and guidance about sharing images with the data guardian for your organisation before embarking widely on this approach.

- Consider obtaining formal written consent from patients that includes specific reference to sharing on social media, the named platforms to be used, the specific visibility of this content to the general public, and the loss of control over these images once shared.

- Consider offering a statement on use of consent somewhere prominent within your social media profile if sharing images on a personal platform.

-Where possible, consider the removal of any potentially identifiable links to the time, place, person (both patient and healthcare professional) and institution as well as the alteration of case specifics such as age and sex if the case history permits.

- Consider that the rarer the clinical case, clinical image or procedure being undertaken, the more identifiable the details of it become.

have adequate information about the benefits and risks of sharing the content. In terms of the UK legal position, Montgomery case law stipulates that patients should be given enough detail about risk whereby "a reasonable person in the patient's position would be likely to attach significance to the risk, or the doctor is or should reasonably be aware that the particular patient would be likely to attach significance to it" ${ }^{\text {. }}$. In the context of social media, discussions on consent should therefore probably encompass the public nature of image distribution and, importantly, the future loss of control of that image once shared. Discussion of image copyright, particularly in an international social media landscape, also probably warrants consideration but is outside the scope of this article.

Whilst the next iteration of healthcare professional guidance is awaited on this subject, reflective professionals can do some simple things to protect their patients' privacy and right to consent whilst also protecting themselves and their reputations. We offer some reflections for practice in BOX 1. Whilst a need could be argued for social media platforms to provide space to attribute consent in the short-hand sharing of medical images, it is unlikely the companies can be compelled to do so. This aspect is perhaps a sphere into which established organisations such as professional societies or medical journals could move and lead by example by sharing such images in a manner compliant with relevant professional standards. In the absence of this step, professional social media users should be mindful of their practice and reflect on how it could be improved. There are already precedents in which healthcare professionals have breached professional standards through their use of social media ${ }^{9}$, both inadvertently and deliberately, resulting in disciplinary action and, in some cases, dismissal.

Social media transcends culture, physical boundaries and international laws, which requires extra consideration when sharing clinical images. Healthcare professionals are encouraged to be reflective practitioners and, as such, it is important that clinicians themselves weigh up the benefits and risks of posting medical images. In doing so, clinicians should be mindful of how these images might be perceived by the patient themselves alongside other professionals and lay people alike. Ultimately, clinicians must always ensure that the patient's trust in our ability to maintain their confidentiality is never compromised through our social media activities.

1. Kennedy, N. A et al. Organisational changes and challenges for inflammatory bowel disease services in the UK during the COVID-19 pandemic. Frontline Gastroenterology 11, 343-350 (2020).

2. Wilcha, R.-J. Effectiveness of virtual medical teaching during the COVID-19 crisis: systematic seview. JMIR Med. Educ. 6, e20963 (2020).

3. Walradt, T., Bilal, M., Wadhwa, V., Chiang, A. L. \& Berzin, T. M. Confidentiality and conflicts of interest. Am. J. Gastroenterol. https://doi.org/10.14309/ajg.0000000000001238 (2021).

4. U.S. Department of Health \& Human Services. Summary of the HIPAA Privacy Rule. HHS.gov. https://www.hhs.gov/hipaa/ for-professionals/privacy/laws-regulations/index.html. (2013)

5. General Medical Council. Doctors use of social media. GMC. Available at: https://www.gmc-uk.org/ethical-guidance/ethicalguidance-for-doctors/doctors-use-of-social-media/doctors-useof-social-media (2013)

6. Department of Health. Information: To Share or not to Share. UK Government https://www.gov.uk/government/publications/ the-information-governance-review (2013).

7. Nature Portfolio. Editorial policies. Nature Portfolio https://www. nature.com/nature-portfolio/editorial-policies (2021).

8. Chan, S. W. et al. Montgomery and informed consent: where are we now? BMJ 357, j2224 (2017).

9. Rimmer, A. Over 1200 NHS staff have been disciplined for social media use. BMJ 362, k3947 (2018).

\section{Acknowledgements}

R.H. is supported by an NHS Research Scotland Career Researcher Fellowship. The authors would like to thank M. Hansen for her insightful comments on the first draft.

\section{Competing interests}

R.H. has received speaker's fees, conference support and consultancy fees from 4D pharma and Nutricia. J.P.S. has received speaker's fees from Abbvie, Takeda and Janssen. 